\title{
Patterns and clinical significance of cervical lymph node metastasis in papillary thyroid cancer patients with Delphian lymph node metastasis
}

\author{
Guibin Zheng ${ }^{1, *}$, Hua Zhang ${ }^{2,}{ }^{*}$, Shaolong Hao ${ }^{1}$, Chengxin Liu ${ }^{1}$, Jie Xu ${ }^{1}$, Jinyao Ning ${ }^{1}$, \\ Guochang Wu${ }^{1}$, Lixin Jiang ${ }^{1}$, Guojun $\mathbf{L i}^{3,4}$, Haitao Zheng ${ }^{1}$ and Xicheng Song ${ }^{2}$ \\ ${ }^{1}$ Department of Thyroid Surgery, The Affiliated Yantai Yuhuangding Hospital of Qingdao University, Yantai, Shandong \\ Province, 264000, China \\ ${ }^{2}$ Department of Otolaryngology-Head and Neck Surgery, The Affiliated Yantai Yuhuangding Hospital of Qingdao University, \\ Yantai, Shandong Province, 264000, China \\ ${ }^{3}$ Departments of Head and Neck Surgery, The University of Texas MD Anderson Cancer Center, Houston, TX 77030, U.S.A. \\ ${ }^{4}$ Department of Epidemiology, The University of Texas MD Anderson Cancer Center, Houston, TX 77030, U.S.A. \\ "These authors contributed equally to this work and are considered co-first authors \\ Correspondence to: Haitao Zheng, email: zhenghaitao1972@126.com \\ Xicheng Song, email: songxicheng@126.com
}

Keywords: Delphian lymph node, papillary thyroid cancer, central neck node metastases, lateral neck node metastases

Received: February 22, $2017 \quad$ Accepted: June 19, $2017 \quad$ Published: July 06, 2017

Copyright: Zheng et al. This is an open-access article distributed under the terms of the Creative Commons Attribution License 3.0 (CC BY 3.0), which permits unrestricted use, distribution, and reproduction in any medium, provided the original author and source are credited.

\section{ABSTRACT}

Although the roles of Delphian lymph node (DLN) metastasis in papillary thyroid cancer (PTC) have been previously reported, there are still limited data on correlations of clinicopathologic factors with DLN metastasis and unique patterns of cervical node subsite metastasis in PTC patients with DLN metastasis. We retrospectively reviewed medical records of $\mathbf{3 2 0}$ patients with a diagnosis of PTC who underwent primary surgery. Clinicopathologic features and DLN metastasis patterns were analyzed for predicting extensive cervical lymph node metastasis. Both univariate and multivariate Cox regression analyses were used to identify independent factors for cervical lymph node metastasis. DLN metastasis was significantly associated with multifocality, tumor size $>1 \mathrm{~cm}$, extrathyroid extension, BRAF ${ }^{\mathrm{V} 600 \mathrm{E}}$ mutation, central neck node metastasis (CNNM), and lateral neck nodes metastases. Patients with DLN metastasis had more lymph node metastases in the central compartment. CNNM number and tumor size $>1 \mathrm{~cm}$ were independent risk factors for DLN metastasis. DLN metastasis was highly predictive of lateral lymph node metastasis with moderate sensitivity and high specificity. DLN metastasis is associated with several poor prognostic factors, including extensive cervical lymph node metastasis, and can serve as a predictor of advanced PTC. The presence of DLN metastasis should prompt surgeons to perform an aggressive surgery approach.

\section{INTRODUCTION}

Central neck nodes (CNNs; also known as level VI nodes), which comprise the Delphian (prelaryngeal), pretreacheal, and paratracheal node groups, are the most common harbors of nodal metastasis in patients with papillary thyroid cancer (PTC). The percentage of PTC patients with central neck node metastasis (CNNM) at primary diagnosis ranges from $20 \%$ to $53.7 \%$ [1-6].
Although some authors have reported that CNNM has no major impact on the survival rate of low-risk PTC patients [3, 7], emerging evidence suggests that CNNM has an adverse effect on the locoregional recurrence and survival rates of intermediate- and high-risk patients $[2,4,8]$. Hence, prophylactic central neck dissection (CND) of the affected side should be performed following thyroidectomy in intermediate- and high-risk PTC patients, as it may improve survival by reducing 
locoregional recurrence and facilitating accurate disease staging [9-11]. However, given the high incidence of laryngeal nerve palsy and hypoparathyroidism following bilateral CND, whether these patients should also have contralateral CND is still a debated issue.

Currently, lateral node metastasis is received more attention. And lateral neck dissection (LND) is recommended for therapeutic purposes. In fact, PTC patients have been reported to have a high frequency of occult lateral node metastasis $[12,13]$. However, the indications for prophylactic LND in PTC patients are controversial and remain unclear, as data for finding an optimal balance between its complications and benefits are limited.

Disease involvement of the Delphian lymph node (DLN), which is located anterior to the cricothyroid membrane, between the cricothyroid muscles (Figure 1), is a poor prognostic predictor in many malignant neck cancers [14, 15]. Thus, DLN status has important implications in planning appropriate surgical treatment and determining outcome. To our knowledge, only 8 studies have investigated the role of DLN in thyroid cancer management [16-23]. With the exception of extensive cervical lymph node metastasis, the clinicopathologic factors these studies report to be associated with DLN metastasis are inconsistent. However, owing to the limited data on DLN metastasis in PTC, its relationship with other nodal metastasis in the central and lateral compartments and its clinical significance are still debated. Hence, the aim of the present study was to assess the risk factors for DLN metastasis and investigate the pattern of cervical LNM to provide information for lymph node dissection in PTC patients with DLN metastasis.

\section{RESULTS}

Of the 320 patients included in the study, 157 (49.1\%) had CNNM, and $4(1.3 \%)$ had DLN metastasis without metastasis to other central compartments. The DLN was detected in 206 patients (64.4\%), among whom, 42 patients $(20.4 \%)$ had confirmed DLN metastasis (Table 1). The percentage of DLN metastasis in patients with PTC was $13.1 \%$. Thyroid follicular tissue was detected in 71 patients $(22.2 \%)$ and the remaining showed with fibro-adipose tissue. One patient had PTC in the prelaryngeal region without any tumor foci in the thyroid gland and was excluded from the analysis.

The univariate analysis revealed that, compared with patients without DLN metastasis, those with DLN metastasis had significantly higher rates of multifocality (47.6\% vs. $28.0 \%, P=0.015)$, tumor size $>1 \mathrm{~cm}$ (71.4\% vs. $31.1 \%, P<0.001)$, ETE $(59.5 \%$ vs. $37.2 \%$, $P=0.009)$, BRAF ${ }^{\mathrm{V} 600 \mathrm{E}}$ mutation $(64.3 \%$ vs. $47.6 \%, P=$ $0.026)$, paratracheal LNM ( $81.0 \%$ vs. $31.3 \%, P<0.001)$, pretracheal LNM $(64.3 \%$ vs. $23.2 \%, P<0.001)$, and CNNM $(90.5 \%$ vs. $41.5 \%, P<0.001)$ and significantly more CNNMs ( $4.3 \pm 3.0$ vs. $2.3 \pm 1.5, P<0.001)$. (Table 2$)$.
The multivariate logistic analysis revealed that, compared with patients without DLN metastasis, those with DLN metastasis had significantly higher rates of tumor size $>1 \mathrm{~cm}(71.4 \%$ vs. $31.1 \%, P=0.008)$ and significantly more paratracheal LNM $(3.2 \pm 2.1$ vs. 1.8 $\pm 1.1, P<0.001)$ and CNNM $(4.3 \pm 3.0$ vs. $2.3 \pm 1.5$, $P<0.001$ ). (Table 3).

Thirty patients underwent modified neck dissection of $\geq 1$ lateral node sublevel. Skip metastases (positive LNN but negative CNN) were detected in 4 of 27 patients (15\%) without DLN metastasis and 1 of 18 patients $(6 \%)$ with DLN metastasis. LNNM was found in 14 of 14 patients (100\%) with DLN metastasis and 4 of 7 patients $(57 \%)$ without DLN metastasis, a significant difference $(P=0.029)$. Patients with DLN metastasis and LNNM had more CNNM than patients without DLN metastasis but with LNNM did. (Table 4).

Of the 14 patients with DLN metastasis who underwent LND, 11 (79\%) had multiple levels with nodal metastasis. Level III was the most frequently involved (86\%), followed by level IV (71\%), and level II (43\%). Only 2 of these patients (14\%) had metastases in level V nodes. (Table 5).

Compared with patients without DLN metastasis, patients with DLN metastasis had more CNNMs $(4.3 \pm$ 3.0 vs. $2.3 \pm 1.5, P<0.001$ ) (Table 2) and were 14 times more likely to have further CNNM. (Table 6).

The incidence of ipsilateral paratracheal LNM and contralateral paratracheal LNM among patients with DLN metastasis (100\% and 50\% separately) were higher than that among patients without DLN metastasis ( $45.5 \%$ and $27.3 \%$ separately).The difference of ipsilateral paratracheal LNM was statistically significant $(P=$ 0.043 ), while the contralateral paratracheal LNM was not $(P=0.6)$. (Table 7).

Bilateral PTC was observed in $14(60.9 \%)$ of DLN-positive patients and 25(34.7\%) of DLN-negative patients, and the difference was significant $(P=0.026)$. Contralateral tumor foci without suspicion preoperatively by US were identified in $9(39.1 \%)$ of DLN-positive patients, and 12(16.7\%) of DLN-negative patients, which had significant difference $(P=0.024)$. The tumor sizes of two groups were too small and no significant difference was found. (Table 8).

Serum thyroglobulin $(\mathrm{Tg})$ of the patients with total thyroidectomy was reviewed after surgery for recurrence monitoring. The median of follow-up was 14 months (range: 8-22 months) and 11 months (range: 1-26 months) for DLN-positive patients and DLN-negative patients, respectively. Unstimulated $\mathrm{Tg} \geq 1 \mathrm{ng} / \mathrm{ml}$ was chosen to evaluate the likelihood of having persistent or recurrent disease in response to an initial surgery, since an unstimulated postoperative $\mathrm{Tg}<1 \mathrm{ng} / \mathrm{ml}$ was associated with favor outcomes with recurrence rates $<1 \%$ [24]. Kaplan Meier analysis showed that DLN-positive patients had significantly a higher rate of unstimulated $\mathrm{Tg} \geq 1 \mathrm{ng} / \mathrm{ml}$ 
Table 1: Rates of Delphian lymph node (DLN) detection and metastasis

\begin{tabular}{lc}
\hline \multicolumn{1}{c}{ Variables } & N/total (\%)* \\
\hline DLN detection & $206 / 320(64.4)$ \\
DLN metastasis & $42 / 206(20.4)$ \\
Central neck node metastasis & $157 / 320(49.1)$ \\
Mean no. of DLNs (range) & $1.7(1-6)$ \\
Mean no. of DLN metastases (range) & $1.4(1-4)$ \\
\hline
\end{tabular}

*Unless otherwise indicated.

Table 2: Comparison of clinicopathological characteristics between DLN-positive/negative patients

\begin{tabular}{|c|c|c|c|}
\hline Variable & $\begin{array}{l}\text { No. of DLN-positive } \\
\text { patients }(\%), n=42\end{array}$ & $\begin{array}{l}\text { No. of DLN-negative } \\
\text { patients }(\%), n=164\end{array}$ & $P$ value \\
\hline Age $\geq 45$ years & $17(40.5)$ & $86(52.4)$ & 0.167 \\
\hline \multicolumn{4}{|l|}{ Sex } \\
\hline Female & $29(69.0)$ & $130(79.3)$ & \multirow[t]{2}{*}{0.429} \\
\hline Male & $13(31.0)$ & $34(20.7)$ & \\
\hline Multifocality & $20(47.6)$ & $46(28.0)$ & 0.015 \\
\hline Tumor size $>1 \mathrm{~cm}$ & $30(71.4)$ & $51(31.1)$ & 0.001 \\
\hline ETE & $25(59.5)$ & $61(37.2)$ & 0.009 \\
\hline Thyroiditis & $15(35.7)$ & $58(35.4)$ & 0.966 \\
\hline \multicolumn{4}{|l|}{ Tumor location } \\
\hline Isthmus/upper third & $18(42.9)$ & $76(46.3)$ & \multirow[t]{2}{*}{0.923} \\
\hline Middle/lower third & $24(57.1)$ & $88(53.7)$ & \\
\hline \multicolumn{4}{|l|}{$\mathrm{BRAF}^{\mathrm{V} 600 \mathrm{E}}$ gene } \\
\hline Mutant & $27(64.3)$ & $78(47.6)$ & \multirow[t]{3}{*}{0.026} \\
\hline Wild type & $7(16.7)$ & $18(11.0)$ & \\
\hline Undetected & $8(19.0)$ & $68(41.5)$ & \\
\hline Paratracheal LNM & $34(81.0)$ & $51(31.1)$ & 0.001 \\
\hline Mean no. of paratracheal $\mathrm{LNM} \pm \mathrm{SD}$ & $3.2 \pm 2.1$ & $1.8 \pm 1.1$ & 0.001 \\
\hline Pretracheal LNM & $27(64.3)$ & $38(23.2)$ & 0.001 \\
\hline Mean no. of pretracheal $\mathrm{LNM} \pm \mathrm{SD}$ & $2.0 \pm 1.6$ & $1.6 \pm 0.8$ & 0.142 \\
\hline CNNM & $38(90.5)$ & $68(41.5)$ & 0.001 \\
\hline Mean no. of $\mathrm{CNNM} \pm \mathrm{SD}$ & $4.3 \pm 3.0$ & $2.3 \pm 1.5$ & 0.001 \\
\hline
\end{tabular}

Note: Data are no. of patients (\%) unless otherwise indicated.

DLN: Delphian lymph node; ETE: extrathyroid extension; LNM: lymph node metastases; CNNM: central neck node metastases.

than DLN-negative patients ( $30.4 \%$ vs. $6.9 \%, P=0.011$ ) as shown in Figure 2. Moreover, disease recurrence was detected on follow-up by US and confirmed by FNA or RAI scaning in 3 DLN-positive patients with unstimulated $\mathrm{Tg}>20 \mathrm{ng} / \mathrm{ml}$. The unstimulated Tg levels in other patients with unstimulated $\mathrm{Tg} \geq 1 \mathrm{ng} / \mathrm{ml}$ were all less than $8 \mathrm{ng} / \mathrm{ml}$; and all these patients were disease recurrence-free after treatment. There were no cases for distant metastasis.

\section{DISCUSSION}

The DLN receives lymphatic drainage from the larynx and thyroid, then drains to the lateral neck nodes through the superior thyroid artery. The median size of DLN metastasis deposits is $0.3 \mathrm{~cm}$ [18]; thus, DLN status is difficult to evaluate preoperatively with ultrasonography, computed tomography, or magnetic resonance imaging 
Table 3: Multivariate logistic regression analysis of Delphian lymph node metastasis

\begin{tabular}{lccc}
\hline \multicolumn{1}{c}{ Covariates } & Adjusted odds ratios & $\mathbf{9 5 \%}$ confidence interval & $P$ value \\
\hline Multifocality & 1.1 & $0.4-2.6$ & 0.908 \\
Tumor size $>1 \mathrm{~cm}$ & 3.5 & $1.4-8.7$ & 0.008 \\
ETE & 1.6 & $0.6-3.9$ & 0.326 \\
BRAF & 0.7 & $0.2-2.4$ & 0.556 \\
No. of paratracheal LNM & 2.0 & $1.4-2.8$ & 0.001 \\
No. of pretracheal LNM & 1.4 & $0.9-2.1$ & 0.217 \\
No. of CNNM & 1.7 & $1.4-2.1$ & 0.001 \\
\hline
\end{tabular}

ETE: extrathyroid extension; LNM: lymph node metastases; CNNM: central neck node metastases.

$[15,22]$. Therefore, DLN status must be assessed by frozen section biopsy intraoperatively to provide information about whether further CND is warranted. DLN excision can be easily performed before thyroidectomy and does not prolong the time of operation or hamper other nodal dissection in the central compartment. It has been reported that DLN metastasis can be used to predict extensive LNM, recurrence, and shorter survival in patients with laryngeal, glottis, or hypopharyngeal cancer [14, 15]. Although the DLN is the first lymph node encountered and could be easily resected during thyroidectomy, whether the DLN can have the same role in thyroid cancer patients is still controversial because of insufficient data.

To the best of our knowledge, only 8 previous studies have investigated the significance of DLN status in thyroid cancer patients [16-23]. In these studies, a DLN was detected in $23.0 \%-74.6 \%$ of patients with thyroid cancer, and of those DLNs, $8.2 \%-24.8 \%$ had metastatic disease. In our study of PTC patients, the DLN detection and metastasis rates were $64.4 \%$ and $20.4 \%$, respectively, which are similar to those reported by Kim et al. (74.6\% and $17.2 \%$, respectively) [19]. Our study had a high rate of DLN detection because it enrolled only PTC patients whose initial treatment was surgery.

All 8 previous studies indicated that DLN metastasis is associated with concomitant cervical LNM, but the clinicopathological factors they reported to be associated with DLN metastasis were not consistent. These factors included multifocality $[19,21]$, autoimmune thyroiditis [25], tumor location [20, 22], lymphovascular invasion

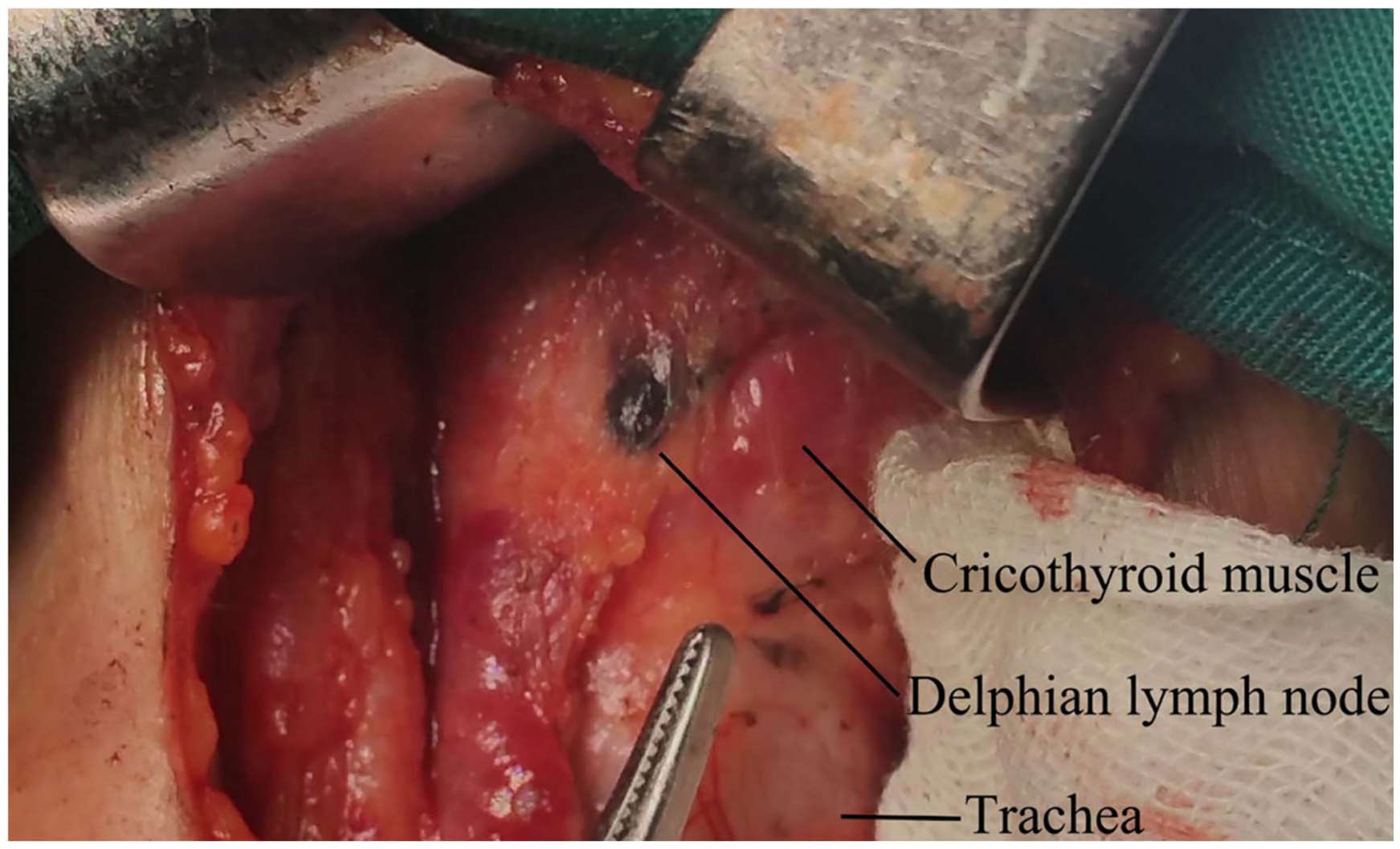

Figure 1: The Delphian lymph node is darkened by nanocarbon. 
Table 4: Clinicopathological characteristics of patients with lateral neck lymph node metastasis (LNM)

\begin{tabular}{|c|c|c|c|}
\hline Variables & $\begin{array}{l}\text { No. of DLN-positive } \\
\text { patients }(\%), n=14\end{array}$ & $\begin{array}{l}\text { No. of DLN-negative patients } \\
\qquad(\%), n=4\end{array}$ & $P$ value \\
\hline Mean no. of LNN removed \pm SD & $19.8 \pm 12.1$ & $18.3 \pm 2.2$ & 0.121 \\
\hline Mean no. of $\mathrm{LNNM} \pm \mathrm{SD}$ & $4.1 \pm 1.6$ & $4.3 \pm 2.6$ & 0.227 \\
\hline \multicolumn{4}{|l|}{$\mathrm{BRAF}^{\mathrm{V} 600 \mathrm{E}}$ gene } \\
\hline Mutant & $10(71)$ & $3(75)$ & 0.887 \\
\hline Wild type & $4(29)$ & $1(25)$ & \\
\hline ETE & $11(80)$ & $4(100)$ & 0.196 \\
\hline Mean tumor size $\pm \mathrm{SD}$, cm & $2.0 \pm 0.9$ & $1.4 \pm 0.5$ & 0.208 \\
\hline Multifocality & $8(57)$ & $4(100)$ & 0.245 \\
\hline Mean no. of CLN removed \pm SD & $7.7 \pm 4.3$ & $9.3 \pm 4.6$ & 0.540 \\
\hline Mean no. of central LNM $\pm \mathrm{SD}$ & $6.0 \pm 3.1$ & $2.3 \pm 2.6$ & 0.044 \\
\hline
\end{tabular}

Note: Data are no. of patients (\%) unless otherwise indicated.

DLN: Delphian lymph node; LNN: lateral neck node; LNNM: lateral neck node metastasis; ETE: extrathyroid extension; CLN: central lymph node; LNM: lymph node metastasis.

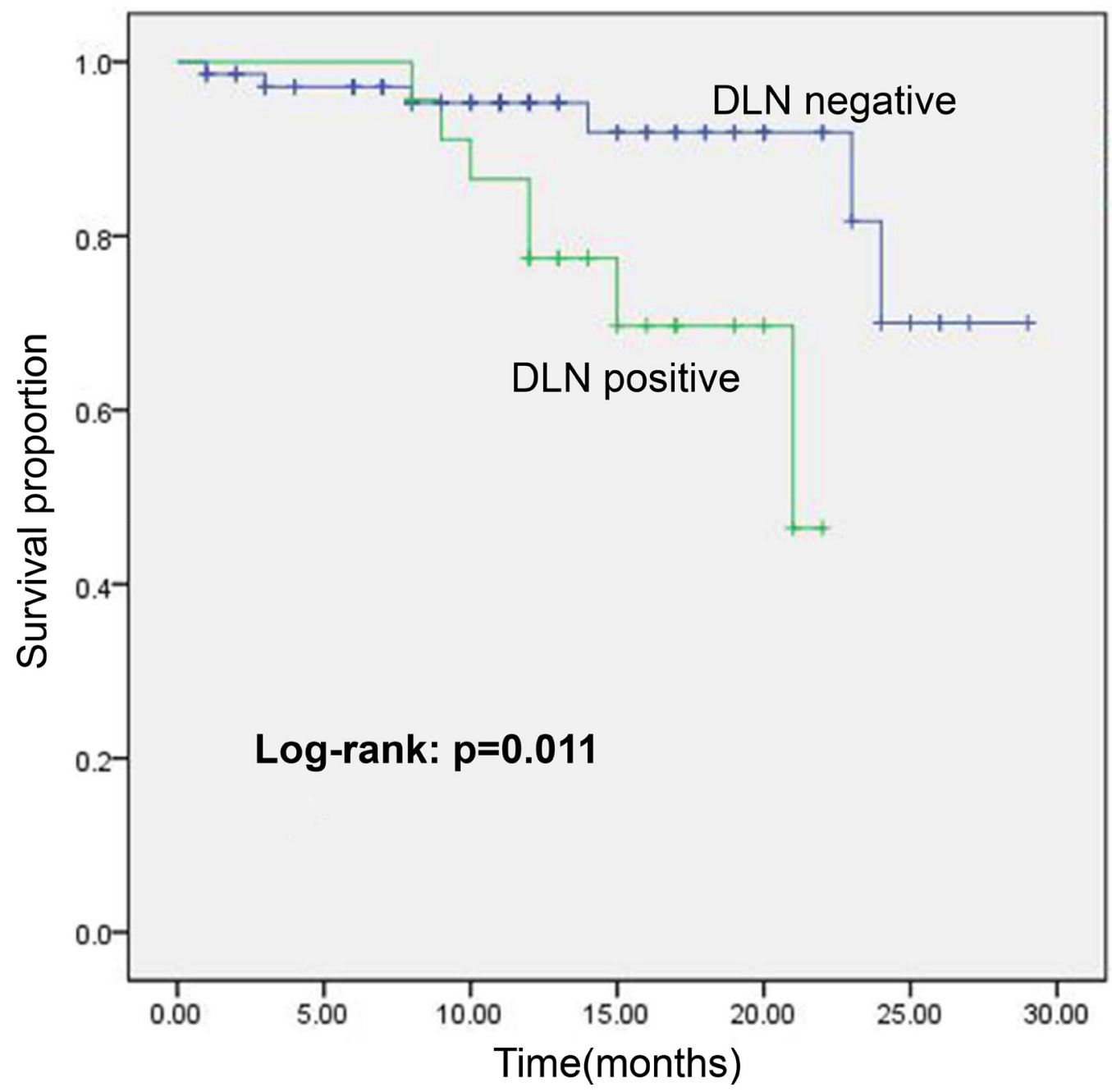

Figure 2: Kaplan Meier plot showing cumulative survival of $\mathrm{Tg} \geq 1 \mathrm{ng} / \mathrm{ml}$ in DLN-positive/-negative patients with total thyroidectomy in the follow-up period. 
Table 5: Metastasis pattern in 14 patients with Delphian lymph node metastasis and lateral neck metastasis

Lateral neck sublevel

No. of patients with positive nodes $(\%)$

\begin{tabular}{lc}
\hline Level II, upper jugular & $6(42.8)$ \\
Level III, middle jugular & $12(85.7)$ \\
Level IV, lower jugular & $10(71.4)$ \\
Level V, posterior triangle & $2(14.3)$ \\
Multiple levels with nodal metastasis & $11(78.6)$ \\
\hline
\end{tabular}

Table 6: Ability of Delphian lymph node metastasis to predict paratracheal, pretracheal, central, and lateral lymph node metastasis (LNM)

\begin{tabular}{lcccccc}
\hline \multicolumn{1}{c}{ LNM types } & Sensitivity $(\mathbf{\%})$ & $\begin{array}{c}\text { Specificity } \\
\mathbf{( \% )}\end{array}$ & PPV (\%) & NPV (\%) & LR+ & LR- \\
\hline Paratracheal & 37.0 & 93.4 & 81.0 & 66.1 & 5.6 & 0.7 \\
Pretracheal & 41.5 & 89.4 & 64.3 & 76.8 & 3.9 & 0.7 \\
Central & 55.8 & 96 & 90.5 & 58.5 & 14.0 & 0.2 \\
Lateral & 77.8 & 100 & 100 & 21.4 & $77.8^{*}$ & $0.8^{*}$ \\
\hline
\end{tabular}

LNM: lymph node metastasis; PPV: Positive predictive value; NPV: Negative predictive value; LR+: positive likelihood radio; LR-: negative likelihood radio.

*Specificity assigned as a value of 0.99 to permit calculation of LR+ and LR-.

Table 7: Ipsilateral or contralateral paratracheal lymph node metastasis (LNM) in DLN-positive/ negative patients

\begin{tabular}{lccc}
\hline \multicolumn{1}{c}{ LNM types } & $\begin{array}{c}\text { No. of DLN-positive patients (\%), } \\
\boldsymbol{n}=\mathbf{6}\end{array}$ & $\begin{array}{c}\text { No. of DLN-negative } \\
\text { patients (\%), } \boldsymbol{n}=\mathbf{1 1}\end{array}$ & $\boldsymbol{P}$ value \\
\hline Ipsilateral paratracheal & $6(100)$ & $5(46)$ & 0.043 \\
Contralateral paratracheal & $3(50)$ & $3(27)$ & 0.600 \\
\hline
\end{tabular}

LNM: lymph node metastasis; DLN: Delphian lymph node.

$[19,22]$, ETE [18-20, 22, 23], larger tumor size [16, 19-23], and cervical LNM [16-23]. Four of the eight previous studies included multivariable analyses, which identified CNNM [19, 21, 22], lymphovascular invasion [19], and tumor location in the isthmus or upper third of the thyroid [22], larger tumor size [21], and ETE [23] as independent risk factors for DLN metastasis. In addition, we investigated for the first time the relationship between DLN metastasis and $\mathrm{BRAF}^{\mathrm{V} 600 \mathrm{E}}$ mutation, a risk factor for neck lymph node metastasis and poor outcome [26]. Our results showed that DLN metastasis is correlated with $\mathrm{BRAF}^{\mathrm{V} 600 \mathrm{E}}$ mutation (correlation coefficient $=0.180$, $P<0.01$ ), although the odds ratio for $\mathrm{BRAF}^{\mathrm{V} 600 \mathrm{E}}$ mutation in the multivariable analysis was 0.7 , which may have been due to the great differences in the sample sizes and $\mathrm{BRAF}^{\mathrm{V} 600 \mathrm{E}}$ mutation detection rates of the groups with or without DLN metastasis. Moreover, compared with patients without DLN metastasis, patients with DLN metastasis had more CNNMs $(P<0.001)$ and were 14 times more likely to have further CNNM. Taken together, these findings indicate that PTC metastasis to the DLN is associated with several poor prognostic factors, including ETE, tumor size $>1 \mathrm{~cm}, \mathrm{BRAF}^{\mathrm{V} 600 \mathrm{E}}$ mutation, and CNNM. According to management guidelines for thyroid cancer, these factors greatly contribute to an intermediate or high risk of disease recurrence [27].

The extent of CND for PTC is still controversial and the exploration of the relationship between DLN metastasis and other CNN subsites would provide important information for surgeon. CNNs are the first nodes to harbor metastatic PTC, and the number of CNNMs is correlated with high recurrence risk and lower overall survival $[4,28]$. In PTC patients, the paratracheal and pretracheal lymph nodes are the most common subsites in the central compartment to harbor metastasis $[6,17,29]$. Our data showed that DLN-positive patients had significantly higher rates of paratracheal LNM $(81.0 \%$ vs $31.1 \%, P<0.001)$ and were 5.6 times more likely to harbor paratracheal LNM than DLN-negative patients. Previous studies showed that, among patients with DLN 
Table 8: Preoperative status of contralateral nodules on US in DLN-positive/-negative patients with total thyroidectomy

\begin{tabular}{|c|c|c|c|}
\hline Variables & $\begin{array}{l}\text { No. of DLN-positive } \\
\text { patients }(\%), n=23\end{array}$ & $\begin{array}{l}\text { No. of DLN-negative patients } \\
\qquad(\%), n=72\end{array}$ & $P$ value \\
\hline Bilateral PTC & $14(60.9)$ & $25(34.7)$ & 0.026 \\
\hline \multicolumn{4}{|c|}{$\begin{array}{l}\text { Preoperative status of contralateral nodules on } \\
\text { US }\end{array}$} \\
\hline Unsuspected & $9(39.1)$ & $12(16.7)$ & 0.024 \\
\hline Tumor size $(\mathrm{cm})^{*}$ & $0.40 \pm 0.17$ & $0.33 \pm 0.20$ & 0.768 \\
\hline
\end{tabular}

US: ultrasonography; DLN: Delphian lymph node

*The size of the contralateral PTC foci that was unsuspected on preoperative US.

metastasis, $42.9 \%-84.6 \%$ have ipsilateral paratracheal LNM, whereas $1.7 \%-9.8 \%$ have contralateral paratracheal LNM $[10,30,31]$. The negative predictive value of not having contralateral paratracheal LNM in the absence of DLN metastasis is $90.3 \%$ [19]. These results suggest that CND on the affected side (including DLN, ipsilateral paratracheal lymph nodes, and pretrecheal lymph nodes) should be routinely performed in DLN-positive patients, while ipsilateral CND is sufficient for DLN-negative patients.

Contralateral CND (including contralateral paratracheal lymph nodes), as part of bilateral CND, can be performed on the premise of total thyroidectomy. In our study, bilateral PTC was observed to have a significant difference between DLN-positive patients and DLN-negative patients. Contralateral tumor foci without suspicion preoperatively by US were significantly higher than that of DLN-negative patients. The tumor sizes of two groups were too small and no significant difference was found. Thus, we recommended that total thyroidectomy should be performed in DLN-positive patients with nodules in contralateral lobe in order to remove all the tumor foci unsuspected preoperatively by US. It was reported that contralateral paratracheal LNM was observed in $13.3 \%-21.0 \%$ of patients with PTC and associated with ipsilateral CNNM and DLN metastasis (P $=0.028)[32,33]$. Kim et al. reported that the incidence of contralateral paratracheal LNM among DLN-positive patients $(41.9 \%)$ is significantly higher than that among DLN-negative patients $(9.6 \% ; P<0.001)$ regardless of tumor size. The same results was observed in PTMC between the two groups $(41.7 \%$ vs $6.6 \%, P<0.001)[19]$. Our data also showed the same tendency ( $50 \%$ vs $27 \%)$, although it had not a significant difference, likely because of the small number of patients with bilateral paratracheal lymph node dissection. It was suggested that it's very likely to suffer from LNM in contralateral paratracheal region when PTC metastasize to DLN. Moreover, the recurrence rate of contralateral paratracheal lymph node with a mean of follow-up of 12.1 years was $1.8 \%$ in patients who received total thyroidectomy and ipsilateral CND at initial surgery [34]. Since bilateral CND, which had decreased CNN recurrence, can be performed safely by experienced surgeons at high-volume centers [35, 36], contralateral CND should be considered in DLN-positive patients with intermediate or high risk of recurrence. If contralateral paratracheal lymph node dissection cannot be performed, such patients' follow-up care should include careful surveillance of the contralateral CNN.

Currently, lateral neck dissection is performed for therapeutic purposes only. Lateral neck dissection should include the removal of at least the level IIa, IIb, III, and IV nodes, as these are the most common locations of metastatic disease, which is found in level III nodes in $57.0 \%-74.8 \%$ of patients, level IV nodes in $51.0 \%-75.9 \%$ of patients, and level II nodes in $52.0 \%-72.2 \%$ of patients $[31,37,38]$. Moreover, $46.1 \%-80.7 \%$ of patients have lateral nodal disease at multiple levels [31, 38]. Previous studies have shown that DLN metastasis is a risk factor for lateral nodal metastasis [16-19]. In the present study, of the 42 patients with DLN metastasis, 14 (33\%) had preoperative evidence of lateral node involvement, and metastatic disease to lateral nodes at multiple levels was observed in $11(78.6 \%)$ DLN-positive patients with LNNM. These results indicate that DLN metastasis is associated with extensive nodal metastasis in the lateral neck compartment and that patients with DLN and lateral node metastasis should undergo careful LND. The relationship between DLN and occult LNNM in patients with PTC has not been discussed yet. There are no clear indications for performing LND in patients with no preoperative evidence of LNNM. Fraser et al. reported that 41 of 137 PTC patients (30\%) had occult disease in just level III [13]. Moreover, Mazzaschi et al. found that 32 of 40 patients $(80 \%)$ with differentiated thyroid cancer who did not have preoperative evidence of cervical LNM had ipsilateral CNNM and LNNM simultaneously [29]. These studies highlight the great discrepancy between the high frequency of pathological LNNM and low rate of clinical lateral nodal involvement. Other studies have reported that DLN metastasis is highly predictive of LNNM [16-19], which is consistent with our results. Several of those studies reported that patients with DLN metastasis are 3.5-8.8 times more likely than patients without DLN 
metastasis to have LNNM [16-18]. In addition, because patients with DLN metastasis have extensive CNNM, it is reasonable to assume that a number of patients with DLN metastasis have occult LNNM. Hence, prophylactic LND may benefit patients with DLN metastasis by reducing the risk of regional recurrence and improving survival $[39,40]$. Given the significant morbidity of LND, patients with DLN metastasis but no evidence of lateral nodal involvement may benefit from sentinel node biopsy, which has demonstrated promising results [41, 42], for evidence of occult metastasis and justification of LND.

Several limitations of our study should be considered. First, the relationship between DLN and lymphovascular invasion was not evaluated because this information cannot obtained from the pathology reports of the patients, which lead to a loss of reference in the analyses of the relationship between DLN metastasis and other nodal metastasis, although only 2 studies reported an association between DLN metastasis and lymphovascular invasion [19, 22]. Second, the sample size of cases underwent bilateral CND and LND is small, which may cause a bias in our results. As PTC is an indolent tumor and short-time follow-up could not get enough information, further studies with larger patient population and longer follow-up period are warrant to justify the clinical significance of DLN metastasis.

In conclusion, our findings demonstrate that once PTC has metastasized to the DLN, the disease has become aggressive, with several poor prognostic factors that predict an intermediate or even high risk of recurrence. Because patients with DLN metastasis have extensive CNNM and a high risk of LNNM, such patients' DLNs should be assessed intraoperatively with frozen section biopsy which may help determine an aggressive surgery approach.

\section{MATERIALS AND METHODS}

\section{Study patients}

We retrospectively reviewed the medical records of 320 patients with a final diagnosis of PTC at primary surgery performed between August 2014 and May 2016 in the Thyroid Surgery Department and Otorhinolaryngology-Head \& Neck Surgery Department at Qingdao University Affiliated Yantai Yuhuangding Hospital, China.

The study protocol was approved by the Committee of Ethics in Research of Yuhuangding Hospital of Qingdao University. All of the following procedures were in accordance with the ethical standards of the responsible committees for human experimentation (institutional and national) and with the Helsinki Declaration of 1975, as revised in 2008 .

Tissues with DLNs were excised in all 320 cases, then were labeled as DLNs and examined by separate histopathological examinations. DLNs were detected in 206 patients, and DLN metastasis was confirmed in 42 of these patients. We investigated the relationship between DLN metastasis and gender, age, tumor size, multifocality, thyroiditis, $\mathrm{BRAF}^{\mathrm{V} 600 \mathrm{E}}$ mutation, extrathyroid extension (ETE), tumor location, CNNM, and lateral neck node metastasis (LNNM). The central neck lymph node classification was assigned, except DLN, to investigate the relationship with further metastasis in other central subsites.

\section{Surgery}

All patients underwent typical thyroid dissection. Total or near-total thyroidectomy was performed for bilateral PTC in 155 patients. Unilateral thyroidectomy plus isthmectomy were performed for unilateral PTC in 175 patients, and subtotal thyroidectomy was added if benign nodules were found in the contralateral lobe. CND was performed routinely on the affected side. CND on the contralateral side was performed when any of the central lymph nodes were found to be suspicious on preoperative imaging exmaination or upon intraoperative inspection or palpation. LND was performed only if preoperative fine needle aspiration cytology or intraoperative frozen section biopsy revealed evidence of metastasis.

\section{Cervical lymph node removal}

Soft tissue above the thyroid isthmus and anterior to the cricothyroid membrane between the cricothyroid muscles was removed and labeled as the DLN. The remaining central lymph nodes were subdivided into pretracheal, ipsilateral and contralateral paratracheal groups according to their locations. LND was performed to remove the lateral level II, III, IV, and V nodes.

\section{$B R A F^{\mathrm{V} 600 \mathrm{E}}$ mutation detection}

Tumor samples were excised, immediately put into sterile vials containing chilled phosphate-buffered saline $(\mathrm{pH}=7.2)$, sent to the biomolecular laboratory, and frozen at $-80^{\circ} \mathrm{C}$ for molecular investigations.

DNA was extracted with a DNA extraction kit (Promega Corporation, USA), and BRAF gene exon 15 was detected with a BRAF mutant gene detection kit (Amoy Diagnostics Co., LTD, China) and an ABI7500 real-time polymerase chain reaction amplifier (Applied Biosystems, USA). For the amplification of exon 15 of BRAF, the forward primer 5'-TCATAATGCTTGCTCTGA TAGGA-3' and reverse primer 5'-GGCCAAAAATTT AATCAGTGGA-3' were used. All BRAF ${ }^{\mathrm{V} 600 \mathrm{E}}$ mutation detection procedures and results analysis were conducted by technicians in our institution's biomolecular laboratory.

\section{Statistical analysis}

SPSS v. 18.0 (SPSS, Inc., Chicago, IL, USA) was used for all statistical analyses. Categorical and continuous 
data were tested with the Student $t$-test, chi-square test, or Fisher exact test. Variables found to be significantly different between groups in the univariate analysis were included in the multivariate logistic regression analysis. Differences were considered significant when $P<0.05$.

\section{Abbreviations}

DLN: Delphian lymph node; PTC: papillary thyroid cancer; CNNM: central neck node metastases; CNNs: Central neck nodes; CND: central neck dissection; LND: lateral neck dissection; ETE: extrathyroid extension; LNNM: lateral neck node metastases.

\section{Authors' contributions}

G.B.Z., H.Z., X.C.S. and H.T.Z. conceived and developed the methodology; S.L.H., C.X.L. and G.L. performed clinicopathological data acquisition and their statistical analysis; J.X., H.Z., J.Y.N., G.C.W. and L.X.J. performed clinical and surgical evaluation. All the coauthors discussed the results with a major contribution of G.B.Z., H.Z., X.C.S. and H.T.Z. The overall study was supervised by G.L., X.C.S. and H.T.Z.

\section{CONFLICTS OF INTEREST}

The authors have no conflicts of interest to declare.

\section{FUNDING}

This work was supported by the Natural Science Fund of Shandong Province, China (No: ZR2015HL092); Key Research Project of Shandong Province, China (No: 2015GSF118090); Human Resources and Social Security Department of Shandong Province, China (No: 2013528); and Yantai Municipal Scientific and Technological Development Project (No: 2015YT06000060).

\section{REFERENCES}

1. Nam-Goong IS, Kim HY, Gong G, Lee HK, Hong SJ, Kim WB, Shong YK. Ultrasonography-guided fine-needle aspiration of thyroid incidentaloma: correlation with pathological findings. Clin Endocrinol. 2004; 60:21-28.

2. Chow SM, Law SC, Chan JK, Au SK, Yau S, Lau WH. Papillary microcarcinoma of the thyroid-Prognostic significance of lymph node metastasis and multifocality. Cancer. 2003; 98:31-40.

3. Ito Y, Uruno T, Nakano K, Takamura Y, Miya A, Kobayashi K, Yokozawa T, Matsuzuka F, Kuma S, Kuma K. An observation trial without surgical treatment in patients with papillary microcarcinoma of the thyroid. Thyroid. 2003; 13:381-387.

4. Adam MA, Pura J, Goffredo P, Dinan MA, Reed SD, Scheri RP, Hyslop T, Roman SA, Sosa JA. Presence and number of lymph node metastases are associated with compromised survival for patients younger than age 45 years with papillary thyroid cancer. J Clin Oncol. 2015; 33:2370-2375.

5. Ito $\mathrm{Y}$, Jikuzono T, Higashiyama T, Asahi S, Tomoda C, Takamura Y, Miya A, Kobayashi K, Matsuzuka F, Kuma K, Miyauchi A. Clinical significance of lymph node metastasis of thyroid papillary carcinoma located in one lobe. World J Surg. 2006; 30:1821-1828.

6. Jiang LH, Chen C, Tan Z, Lu XX, Hu SS, Wang QL, Hou XX, Cao J, Ge MH. Clinical Characteristics Related to Central Lymph Node Metastasis in cN0 Papillary Thyroid Carcinoma: A Retrospective Study of 916 Patients. Int J Endocrinol. 2014; 2014:385787.

7. Zaydfudim V, Feurer ID, Griffin MR, Phay JE. The impact of lymph node involvement on survival in patients with papillary and follicular thyroid carcinoma. Surgery. 2008; 144:1070-1077.

8. Tran Cao HS, Johnston LE, Chang DC, Bouvet M. A critical analysis of the American Joint Committee on Cancer (AJCC) staging system for differentiated thyroid carcinoma in young patients on the basis of the Surveillance, Epidemiology, and End Results (SEER) registry. Surgery. 2012; 152:145-151.

9. Popadich A, Levin O, Lee JC, Smooke-Praw S, Ro K, Fazel M, Arora A, Tolley NS, Palazzo F, Learoyd DL, Sidhu S, Delbridge L, Sywak M, Yeh MW. A multicenter cohort study of total thyroidectomy and routine central lymph node dissection for cN0 papillary thyroid cancer. Surgery. 2011; 150:1048-1057.

10. Roh JL, Kim JM, Park CI. Central lymph node metastasis of unilateral papillary thyroid carcinoma: patterns and factors predictive of nodal metastasis, morbidity, and recurrence. Ann Surg Oncol. 2011; 18: 2245-2250.

11. Kim KE, Kim EK, Yoon JH, Han KH, Moon HJ, Kwak JY. Preoperative prediction of central lymph node metastasis in thyroid papillary microcarcinoma using clinicopathologic and sonographic features. World J Surg. 2013; 37:385-391.

12. Lee SK, Lee JH, Bae SY, Kim J, Kim M, Lee HC, Jung YY, Kil WH, Kim SW, Lee JE, Nam SJ, Choe JH, Kim JH, Kim JS. Lateral neck sentinel lymph node biopsy in papillary thyroid carcinoma, is it really necessary? A randomized, controlled study. Surgery. 2015; 157:518-525.

13. Fraser S, Zaidi N, Norlen O, Glover A, Kruijff S, Sywak M, Delbridge L, Sidhu SB. Incidence and risk factors for occult level 3 lymph node metastases in papillary thyroid cancer. Ann Surg Oncol. 2016; 23:3587-3592.

14. Ferlito A, Shaha AR, Rinaldo A. Prognostic value of Delphian lymph node metastasis from laryngeal and hypopharyngeal cancer. Acta Otolaryngol. 2002; 122:456-457.

15. Wierzbicka M, Leszczyńska M, Młodkowska A, Szyfter W, Bartochowska A. The impact of prelaryngeal node metastases on early glottic cancer treatment results. Eur Arch Otorhinolaryngol. 2012; 269:193-9.

16. Isaacs JD, Lundgren CI, Sidhu SB, Sywak MS, Edhouse PJ, Delbridge LW. The Delphian lymph node in thyroid cancer. Ann Surg. 2008; 247:477-482. 
17. Isaacs JD, McMullen TP, Sidhu SB, Sywak MS, Robinson BG, Delbridge LW. Predictive value of the Delphian and level VI nodes in papillary thyroid cancer. ANZ J Surg. 2010; 80:834-838.

18. Iyer NG, Kumar A, Nixon IJ, Patel SG, Ganly I, Tuttle RM, Shah JP, Shaha AR. Incidence and significance of Delphian node metastasis in papillary thyroid cancer. Ann Surg. 2011; 253:988-991.

19. Kim WW, Yang SI, Kim JH, Choi YS, Park YH, Kwon SK. Experience and analysis of Delphian lymph node metastasis in patients with papillary thyroid carcinoma. World J Surg Oncol. 2012; 10:226.

20. Lee YC, Shin SY, Kwon KH, Eun YG. Incidence and clinical characteristics of prelaryngeal lymph node metastasis in papillary thyroid cancer. Eur Arch Otorhinolaryngol. 2013; 270:2547-2550.

21. Oh EM, Chung YS, Lee YD. Clinical significance of Delphian lymph node metastasis in papillary thyroid carcinoma. World J Surg. 2013; 37:2594-2599.

22. Chai YJ, Kim SJ, Choi JY, Koo H, Lee KE, Youn YK. Papillary thyroid carcinoma located in the isthmus or upper third is associated with Delphian lymph node metastasis. World J Surg. 2014; 38:1306-1311.

23. Tan Z, Ge MH, Zheng CM, Wang QL, Nie XL, Jiang LH. The significance of Delphian lymph node in papillary thyroid cancer. Asia Pac J Clin Oncol. 2016 Mar 17.https:// doi.org/10.1111/ajco.12480. [Epub ahead of print].

24. Ibrahimpasic T, Nixon IJ, Palmer FL, Whitcher MM, Tuttle RM, Shaha A, Patel SG, Shah JP, Ganly I. Undetectable thyroglobulin after total thyroidectomy in patients with low- and intermediate-risk papillary thyroid cancer-is there a need for radioactive iodine therapy? Surgery. 2012; 152:1096-1105.

25. Ormeci $\mathrm{T}$, Colakogullari $\mathrm{M}$, Orhan I. Importance of Delphian lymph node evaluation in autoimmune thyroiditis: fact or fiction? Pol J Radiol. 2016; 81:72-79.

26. Howell GM, Nikiforova MN, Carty SE, Armstrong MJ, Hodak SP, Stang MT, McCoy KL, Nikiforov YE, Yip L. BRAF V600E mutation independently predicts central compartment lymph node metastasis in patients with papillary thyroid cancer. Ann Surg Oncol. 2013; 20:47-52.

27. Haugen BR, Alexander EK, Bible KC, Doherty GM, Mandel SJ, Nikiforov YE, Pacini F, Randolph GW, Sawka AM, Schlumberger M, Schuff KG, Sherman SI, Sosa JA, et al. 2015 American Thyroid Association Management Guidelines for Adult Patients with Thyroid Nodules and Differentiated Thyroid Cancer: The American Thyroid Association Guidelines Task Force on Thyroid Nodules and Differentiated Thyroid Cancer. Thyroid. 2016; 26:1-133.

28. Leboulleux S, Rubino C, Baudin E, Caillou B, Hartl DM, Bidart JM, Travagli JP, Schlumberger M. Prognostic factors for persistent or recurrent disease of papillary thyroid carcinoma with neck lymph node metastases and/or tumor extension beyond the thyroid capsule at initial diagnosis. $\mathrm{J}$ Clin Endocrinol Metab. 2005; 90:5723-5729.

29. Mazzaschi O, Lefevre M, Angelard B, Chabbertbuffet N, Guily JL, Périé S. Correlation between Central and Lateral
Neck Dissection in Differentiated Thyroid Carcinoma. Int J Otolaryngol. 2012; 01:109-115.

30. Roh JL, Kim JM, Park CI. Central cervical nodal metastasis from papillary thyroid microcarcinoma: pattern and factors predictive of nodal metastasis. Ann Surg Oncol. 2008; 15:2482-2486.

31. Roh JL, Kim JM, Park CI. Lateral cervical lymph node metastases from papillary thyroid carcinoma: pattern of nodal metastases and optimal strategy for neck dissection. Ann Surg Oncol. 2008; 15:1177-1182.

32. Mirallié E, Visset J, Sagan C, Hamy A, Le Bodic MF, Paineau J. Localization of cervical node metastasis of papillary thyroid carcinoma. World J Surg. 1999; 23:970-973.

33. Chen Q, Zou XH, Wei T, Huang QS, Sun YH, Zhu JQ. Prediction of ipsilateral and contralateral central lymph node metastasis in unilateral papillary thyroid carcinoma: a retrospective study. Gland Surg. 2015; 4:288-294.

34. Ohshima A, Yamashita $\mathrm{H}$, Noguchi S, Uchino S, Watanabe S, Toda M, Koike E, Takatu K, Yamashita H. Indications for bilateral modified radical neck dissection in patients with papillary carcinoma of the thyroid. Arch Surg. 2000; 135:1194-1198.

35. Hall CM, Snyder SK, Maldonado YM, Lairmore TC. Routine central lymph node dissection with total thyroidectomy for papillary thyroid cancer potentially minimizes level VI recurrence. Surgery. 2016; 160:1049-1058.

36. Selberherr A, Riss P, Scheuba C, Niederle B. Prophylactic "First-Step" Central Neck Dissection (Level 6) Does Not Increase Morbidity After (Total) Thyroidectomy. Ann Surg Oncol. 2016; 23:4016-4022. w

37. Kupferman ME, Patterson M, Mandel SJ, LiVolsi V, Weber RS. Patterns of lateral neck metastasis in papillary thyroid carcinoma. Arch Otolaryngol Head Neck Surg. 2004; 130:857-860.

38. Zhang XJ, Xu DB, Mu YQ, Chen WK. Should level V be included in lateral neck dissection in treating papillary thyroid carcinoma? World J Surg Oncol. 2013; 25:304.

39. Karakoc D, Ozdemir A. Lymph node surgery in papillary thyroid carcinoma. Int Surg. 2010; 95:142-146.

40. Lee CW, Gong G, Roh JL. Intraoperative diagnosis of central compartment lymph node metastasis predicts recurrence of patients with papillary thyroid carcinoma and clinically node-negative lateral neck and may guide extent of initial surgery. World J Surg. 2015; 39:194-202.

41. Dzodic R, Markovic I, Inic M, Jokic N, Djurisic I, Zegarac M, Pupic G, Milovanovic Z, Jovic V, Jovanovic N. Sentinel lymph node biopsy may be used to support the decision to perform modified radical neck dissection in differentiated thyroid carcinoma. World J Surg. 2006; 30:841-846.

42. Pelizzo MR, Rubello D, Boschin IM, Piotto A, Paggetta C, Toniato A, De Salvo GL, Giuliano A, Mariani G, Casara D. Contribution of SLN investigation with 99mTc-nanocolloid in clinical staging of thyroid cancer: technical feasibility. Eur J Nucl Med Mol Imaging. 2007; 34:934-938. 